\title{
BMJ Open Effects of oligosaccharide-sialic acid (OS) compound on maternal-newborn gut microbiome, glucose metabolism and systematic immunity in pregnancy: protocol for a randomised controlled study
}

\author{
Shuxian Wang, Rui Peng, Shengtang Qin, Yu Liu, Huixia Yang, Jingmei Ma
}

To cite: Wang S, Peng R, Qin S, et al. Effects of oligosaccharidesialic acid (OS) compound on maternal-newborn gut microbiome, glucose metabolism and systematic immunity in pregnancy: protocol for a randomised controlled study. BMJ Open 2019;9:e026583. doi:10.1136/ bmjopen-2018-026583

- Prepublication history for this paper is available online To view these files, please visit the journal online (http://dx.doi. org/10.1136/bmjopen-2018026583).

SW and RP contributed equally.

SW and RP are joint first authors.

Received 11 September 2018 Revised 01 August 2019 Accepted 21 August 2019

\section{Check for updates}

(c) Author(s) (or their employer(s)) 2019. Re-use permitted under CC BY-NC. No commercial re-use. See rights and permissions. Published by BMJ.

OBGYN, Peking University First Hospital, Beijing, China

Correspondence to Dr Jingmei Ma; jingmeima@bjmu.edu.cn

Dr Huixia Yang; yanghuixia@bjmu.edu.cn

\section{ABSTRACT}

Introduction The gut microbiota participates in multiple human biological processes, including metabolism and immune responses. During pregnancy, the dynamics of gut microbiota is involved in physiological adaptation. The disturbed profile of microbiome is associated with maternal complications, such as gestational diabetes mellitus (GDM), which further transfers to the offspring and influence their metabolic and immunological functions in the long term. Prebiotics targeting the gut microbiota and modulating metabolic and immune functions have been shown to be effective in non-pregnant populations with metabolic syndrome. Hence, we propose the use of a prebiotic supplement, oligosaccharide-sialic acid (OS) from the first trimester until delivery in pregnant women, can benefit maternal/new-born gut microbiome, glucose metabolism and innate immunity.

Methods and analysis In this prospective double-blinded randomised clinical trial, recruited singleton pregnancies will be stratified by body mass index (BMI) and randomly assigned to consume the OS preparation or placebo daily from the first trimester. At seven later time points (before and after recruitment in the first trimester, in the middle and third trimesters, before delivery, at birth and 42 days postpartum), compliance will be evaluated and/or biological samples will be collected. Along with maternal clinical information, questionnaires on lifestyle and infant development will be recorded. The primary outcomes are the effect of OS on the maternal-offspring gut microbiome and GDM incidence. The secondary outcomes are maternal glycolipid biochemical parameters, cytokine profiles, weight gain during pregnancy and infant morbidities, growth and development. The study aims to validate the effects of OS on reducing maternal morbidity within different BMI groups. The multiple dimensional dataset generated from the study includes clinical and lifestyle-related information, various biological markers and associated protective or risk factors for morbidity and prognosis. An extended follow-up through 42 days after birth could further explore the intrauterine influence on the long-term health of offspring.

\section{Strengths and limitations of the study}

- This is a prospective randomised clinical trial to validate the oligosaccharide-sialic acid-induced modifications of maternal-neonatal health through gut microbiome beginning in the first trimester.

- The double-blinded and placebo-control design without changing lifestyle, as well as stratification according to body mass index at enrolment improve the objectivity and help reduce bias.

- Multiple dimensional data including clinical parameters and gut microbiota profiles will be collected and analysed comprehensively, which could help to understand the pathophysiological mechanism and intervention target.

- The follow-up period covers the whole gestation and is extended to 42 days postpartum to evaluate the long-term effects.

- It is difficult to fully control all the confounding factors other than diet and exercise that contribute to microbiome profiles.

Ethics and dissemination This protocol has been approved by Peking University First Hospital, National Unit of Clinical Trial Ethics Committee (reference number: 164). The results are expected to be published in scientific manuscripts by 2021.

Trial registration number ChiCTR1800017192.

\section{INTRODUCTION}

Accumulating evidences had shown that human microbiome is associated with immunological function and metabolism. The modified microbiome and activation of the Toll-like receptor-inflammation pathway influenced insulin resistance. ${ }^{1}$ Recent studies have illuminated that both dietary fibre and metformin could alleviate type 2 diabetes by modulating gut microbiome, indicating that 
the gut microbiome is a potential intervention target or prognostic biomarker. ${ }^{23}$

During pregnancy, the maternal gut, vaginal and even oral microbiota change dynamically, constituting physiological adaptations along with changes in hormonal levels, immune responses and metabolism. ${ }^{45}$ Gut microbial perturbance has been found in gestational diabetes mellitus (GDM), preeclampsia and preterm birth. ${ }^{6-8}$ Rosenbaum et al found that structures of gut microbiome were significantly different after stratification by pre-pregnancy body mass index (BMI) ${ }^{9-11}$ Together with plausible microflora originating from the amniotic fluid and placenta, maternal microbiome affected the colonisation and establishment of infant microbiome in early life, which is crucial for development, health and disease susceptibility in the long term. ${ }^{12-14}$ Therefore, maternal gut microbiome profile could be one of key targets to break the vicious cycle and decrease the incidence of complications. Some studies have proved that probiotics were associated with a reduction in GDM incidence and exerted greater benefits in overweight or obese pregnancies ${ }^{1516}$ while some studies did not support this point of view given different genetic backgrounds, durations and objective assessments of the interventions. ${ }^{17} 18$

In contrast to probiotics, composed with active microbiota in food supplements, prebiotics are selectively used by host microorganisms conferring a health benefit, particularly by Lactobacilli and Bifidobacterium. ${ }^{19}{ }^{20}$ Prebiotics can be found in wheat, bananas and onion. ${ }^{21}$ Galacto-oligosaccharides (GOSs), one important component in oligosaccharide-sialic acid (OS), can benefit the maturation of immune system in infants by increasing Bifidobacterium directly ${ }^{22} 23$ and promoting anti-inflammatory factors (IL-10) or reducing proinflammatory cytokines (IL-6, IL-1 $\beta$, TNF- $\alpha$ ) in the elderly. ${ }^{24}{ }^{25}$ The fermentation of GOSs generate physiologically active by-products, such as short-chain fatty acids (SCFAs). SCFAs can target G-protein coupled receptors 41, 43 and histone deacetylase enzymes to reduce systematic inflammation. ${ }^{26-28}$ In clinical trials involving patients with metabolic syndrome, SCFAs entered cholesterol synthesis and lipogenesis pathways to improve lipid profiles. ${ }^{29-31}$ Other mechanisms of gastric emptying, subsequent glucose/insulin disposal or incretin synthesis may also be involved. Sialic acid, a family of nine-carbon acidic monosaccharides, naturally occupies the terminal position of oligosaccharides to perform a synergistic role. ${ }^{32}$ Sialylated human milk oligosaccharides can enhance liver, muscle and brain metabolism by increasing nutrients utilisation among undernutrition infants ${ }^{33}$ and prevent neonatal infection by reducing the adhesion of pathogens to the intestinal epithelium. The observations indicate a potentially health-promoting role of prebiotic to shape the human gut microbiome.

Nonetheless, the beneficial effects of prebiotics during pregnancy remain unclear, or whether there will be different sensitivity to the intervention in high risk pregnancies, such as overweight and obese pregnant women. The aim of this study is to validate the effects of OS on maternal-infant morbidity, and this manuscript presents the protocol of research design, data collection, laboratory methods and statistical analysis plans involved in the project.

\section{METHODS AND ANALYSIS}

\section{Study design, randomisation and blinding}

In this randomised double-blinded, parallel-group clinical study, participants will be allocated into the control and intervention groups at a 1:1 ratio. In the gut microbiota profile, GDM incidence will be compared with illuminate the effects. Metabolic disturbances, immunological responses, other pregnant outcomes, such as preeclampsia and weight gain, as well as the growth and development of offspring in early stages of life will be further compared with validate the clinical significance.

Pregnant women who meet the eligible criteria will be recruited and stratified by BMI according to WHO recommendations for the Asian population ${ }^{34}$ (underweight: $\mathrm{BMI}<18.5 \mathrm{~kg} / \mathrm{m}^{2}$ ) and the Group of China Obesity Task Force of the Chinese Ministry of Health ${ }^{35}$ (overweight: BMI $24-28 \mathrm{~kg} / \mathrm{m}^{2}$, obese: $\mathrm{BMI}>28 \mathrm{~kg} / \mathrm{m}^{2}$ ). From the first trimester (T1) until delivery, participants without any antibiotic exposure in 2 weeks will consume either OS compound or placebo at a dose of $60 \mathrm{~g}$ per day, two times a day (once in the morning and evening) after registration. In order to strengthen adherence, supplements containing three major nutrients, vitamins and minerals will be provided in the form of powder at each follow-up timepoint. The specific compositions of OS compound are galacto-oligosaccharides $(6 \mathrm{~g} / 100 \mathrm{~g})$ and sialic acid $(3 \mathrm{~g} / 100 \mathrm{~g})$ and will only be given to the intervention group. Neonates will be divided into two groups according to mothers' consumption during pregnancy and further subdivided in terms of maternal BMI.

The compliance will be tested by (1) checking the remaining supplements and administration records at each visit, (2) testing the faecal contents of oligosaccharides according to a published method. ${ }^{36}$ Monthly reminders using WeChat (a popular social media in China) will be applied.

The randomised sequences are computer-generated random numbers by biostatistician on 'h6world' platform. After stratification by BMI, participants will be allocated into Group A or B automatically using randomised sequences, and the grouping information will not be available to nurses in advance. The two supplements look similar with a unique code assigned by supplier. Then, nurses will allocate the supplements according to the unique code and grouping information. Both participants and researchers will be blinded to the supplement contents during the whole process. Unblinding will be permissible when (1) interim outcomes are available at 24-28 weeks after the oral glucose tolerance test (OGTT); (2) unexplained adverse reactions occur during the study, such as severe osmotic diarrhoea and (3) participants drop out. 


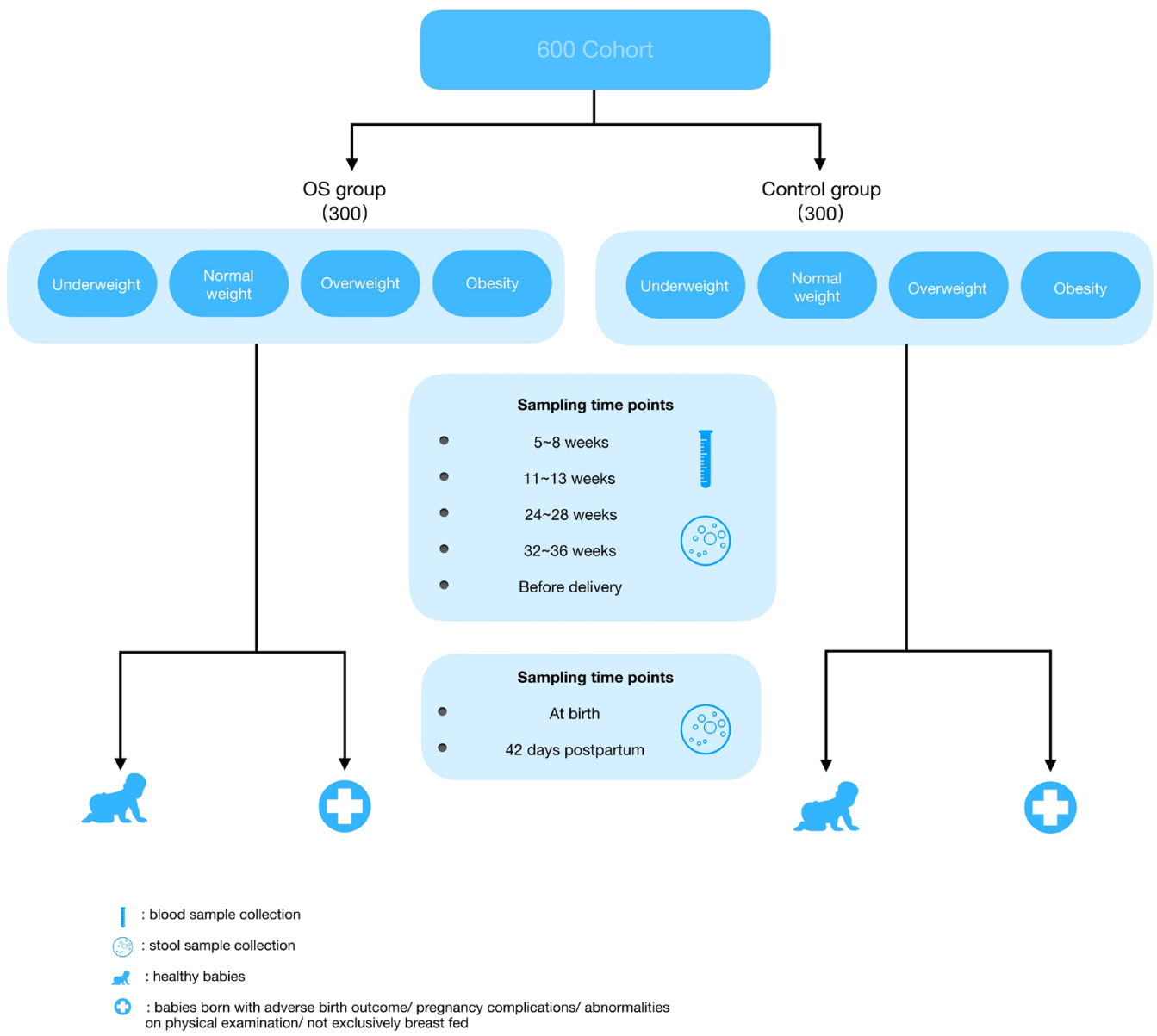

Figure 1 Pregnant women who meet the eligibility criteria at 5-8 weeks of gestation will be recruited and subgrouped based on BMI. Seven timepoints of sample collections will be introduced and informed consent will be signed autonomously after sufficient communication regarding the details of the research. BMI, body mass index; OS, oligosaccharide-sialic acid.

\section{Recruitment}

Peking University First Hospital (PUFH) is a public hospital located in downtown Beijing, China, covering pregnancies with diverse demographic characteristics. The antenatal care provided at PUFH follows national recommendations. All pregnant women will be educated to exclusively breastfeed according to WHO recommendations.

Between 5 and 8 weeks of gestation, for the first prenatal visit, informed consent will be provided autonomously. Sufficient communication will be focused on the details of the research (eligibility criteria, composition, benefits and underlying risks of OS compound) and the process of maternal-fetal/infant follow-up (figure 1). Then, trained nurses will provide a pamphlet with the contents of scientific antenatal care and exclusive suggestions to avoid excessive energy intake or extra prebiotic supplement. Exclusively breastfed (confirmed by questionnaires) will be encouraged. Although only healthy, non-formula-fed infants will be included for further comparison, considering the rate of exclusive breast feeding within 1 month is $66.1 \%,{ }^{37}$ or $70 \%$ after strengthened education, we believe that the sample pool will be large enough for further analysis.
There are over 6000 deliveries per year in PUFH, with proportions of $14.2 \%, 66.3 \%, 14.8 \%$ and $4.7 \%$ for underweight, normal weight, overweight and obese pregnancies, respectively. ${ }^{38}$ To generate a convincing conclusion, recruitment will be initiated in 1 August 2019 and completed on 31 December 2019. If ancillary studies need to be carried out, additional consent provisions will be signed.

\section{Eligibility criteria}

For pregnant women

Inclusion criteria

- 18-40 years of age, residing in Beijing, understanding and willing to sign informed consent.

- Singleton pregnancy.

- First prenatal care visit between 5 and 8 gestational weeks.

\section{Exclusion criteria}

- Smoking, excessive alcohol consumption or drug use.

- Pregnancy complicated with chronic diseases, for example, known pre-existing diabetes, impaired fasting glucose or impaired glucose tolerance, chronic hypertension and so on or taking any prescribed chronic medications, including steroids. 
Table 1 Schematic representation of data and biological sample collection among maternal-neonatal pairs at seven important timepoints

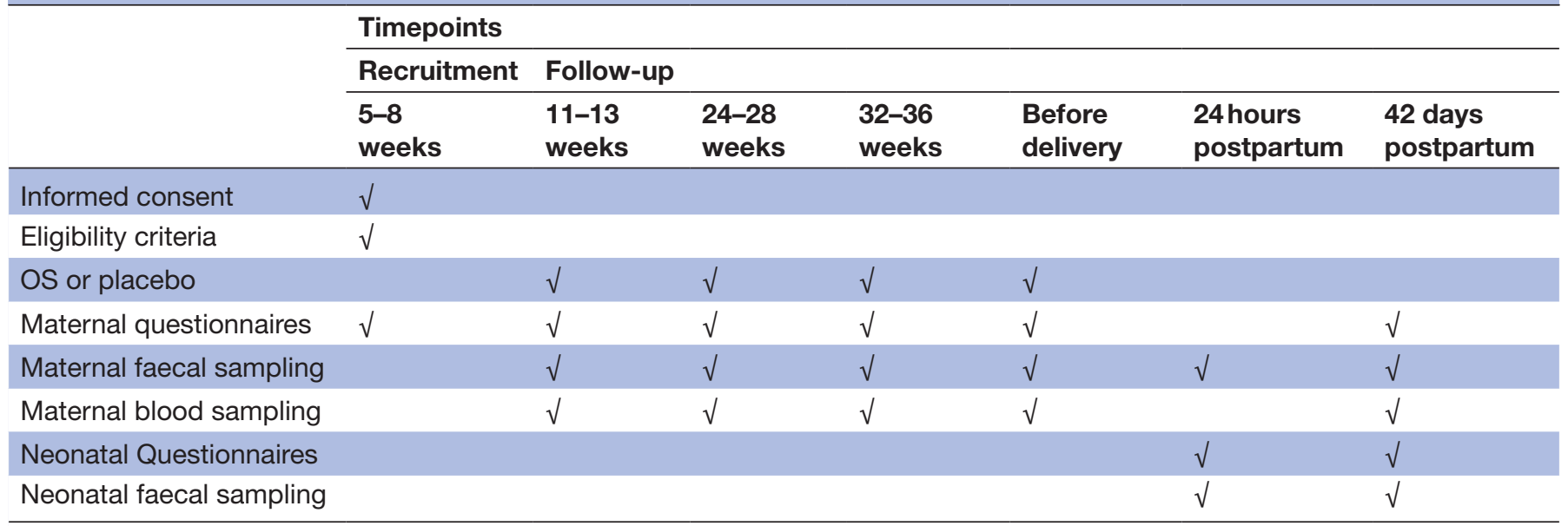

Maternal data will mainly be obtained from questionnaires or medical records and collected at the corresponding pregnancy weeks. Neonatal growth and development conditions will be evaluated by paediatricians and recorded in questionnaires.

Faecal samples and blood samples will be collected at the first five timepoints.

Faecal samples of both mothers and infants will be collected at the last two timepoints.

OS, oligosaccharide-sialic acid.

For new-borns

Inclusion criteria

- Without maternal complications during pregnancy or at delivery.

- Full-term birth weights of 2500-4000 g.

- No genetic or morphological abnormalities.

- Exclusively breastfed.

Termination criteria

Unexplained adverse reactions during the study, such as severe osmotic diarrhoea.

\section{Data and biological sample collection}

The gestational age is determined by last menstrual period and will be rechecked by transvaginal ultrasound in the first trimester. The questionnaires will be filled out and/or collected along with biological samples at 5-8 weeks of gestation (during registration), 11-13 weeks of gestation, 24-28 weeks of gestation, 32-36 weeks of gestation, before delivery, at birth and 42 days postpartum (table 1). To reduce the drop-out rate, supplements will be provided at each timepoint of follow-up. All samples will be collected in sterile tubes and stored at $-80^{\circ} \mathrm{C}$ until testing. All metabolic and immune parameters will be analysed through routine or extra testing. Faecal samples will be collected for gut microbiome analysis.

Relevant information extracted from medical records and questionnaires, including gestational age, delivery mode, birth weight, feeding pattern and adverse pregnancy outcomes, such as GDM, hypertensive disorders complicated in pregnancy, premature labour, spontaneous abortion and macrosomia as well as compliance with the intervention, will be recorded independently and double checked by two well-trained staff. The physical activity level will be measured using the International
Physical Activity Questionnaire (IPAQ) - Short Last 7 Days Self-Administered Format. A 3-day dietary record describing all consumed foods will be collected by MyFitnessPal Software (Under Armour, USA). The collected dataset will be stored using password-protected web-based platforms.

\section{Sample processing and bioassays}

Faecal DNA will be extracted with a commercial clean-up kit (Qiagen, Hilden, Germany) and the V3-V4 region of 16S rRNA gene will be amplified by PCR with the forward primer 5'TCGTCGGCAGCGTCAGATGTGTATAAGA GACAGCCTACGGGNGGCWGCAG and the reverse primer 5'GTCTCGTGGGCTCGGAGATGTGTATAAG AGACAGGACTACHVGGGTATCTAATCC.

The PCR products will be sequenced on Illumina Hiseq 2500 platform, and the sequence data will be filtered, denoised and processed on QIIME software. Based on the Silva database (https://www.arb-silva.de/documentation/release-128/), sequences will be clustered into distinct operational taxonomic units (OTUs) at a $97 \%$ similarity level. The absolute and relative abundance of bacterial OTUs will be classified into several levels: phylum, class, order, family and genus.

Blood samples will be analysed for immunological parameters (Interlukin (IL)-1 $\beta$, IL-6, IL-8, Tumor Necrosis Factor- $\alpha$ (TNF- $\alpha$ ), C-reactive protein (CRP) and IL-10) using multiplex beads array assay for cytokines (Merck Millipore, Merck KGaA, Darmstadt, Germany), for the lipid profile (Triglyceride (TGs), Total cholesterol (TCHO), very low-density lipoprotein (VLDL), low-density lipoproteins (LDL) and high-density lipoproteins (HDL)) using peroxidase (POD) (AU Chemistry System \& Beckman Coulter, USA) and for the glucose metabolism 


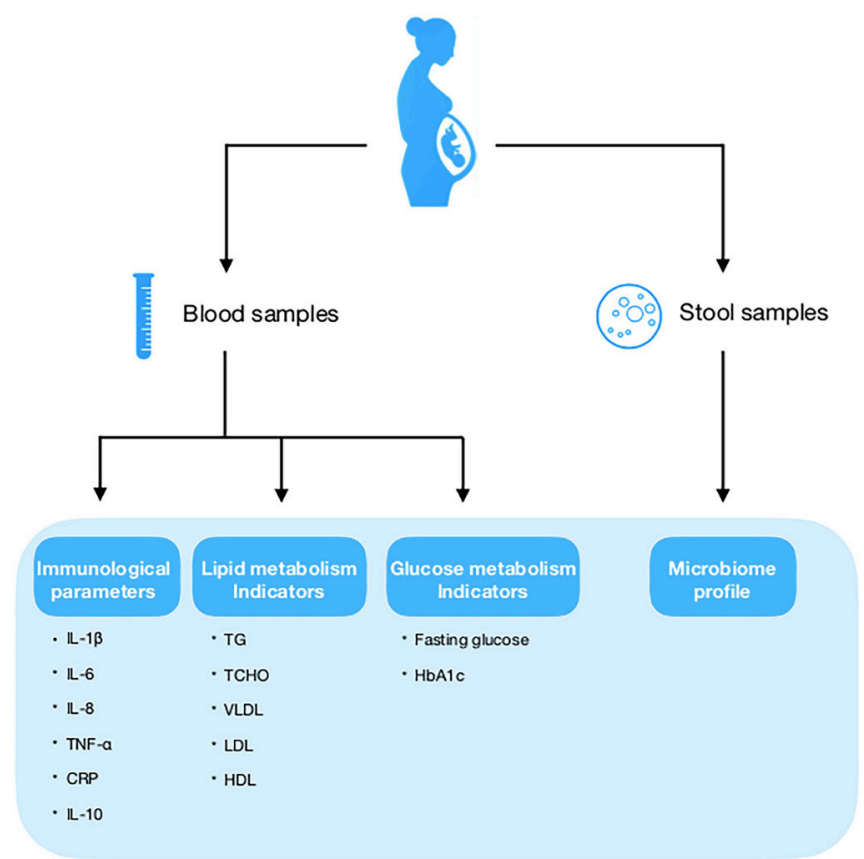

Figure 2 Maternal-neonatal sample schedules during gestation and 42 days postpartum. The faecal samples will be collected for microbiome profile analysis. The blood samples will be collected for glycolipid biochemical parameters and cytokine levels.

index (fasting glucose level, HbA1c and OGTT) using hexokinase (AU Chemistry System \& Beckman Coulter, USA) (figure 2).

\section{Outcome measures}

Primary outcomes

- The effect of OS on maternal-neonatal gut microbiota profiles.

- OTU number.

- alpha diversity (Shannon index or Simpson index).

- beta diversity (weighted or unweighted Unifrac Distance, statistically significant clusters at the genus level).

- The incidence of GDM using the criteria of the International Association of the Diabetes and Pregnancy Study Group (IADPSG $)^{39}$ based on a $75 \mathrm{~g}$ OGTT at 24-28 gestational weeks.

\section{Secondary outcomes}

Maternal

- Glycolipid biochemical parameters.

- Cytokine levels.

- Gestational weight gain.

- Incidence of other complications: preeclampsia, hypertension in pregnancy, preterm labour, spontaneous abortion, caesarean delivery, macrosomia and large for gestational age.

\section{Neonates}

- Birth weight/birth centile.
- Afterbirth growth (circumference of head, chest, biceps, along with weight gain).

Interim outcomes: effect and safety evaluation after OGTT

- Obstetric complications.

- Medical complications.

- Adverse reactions.

\section{Statistical plan}

Power and sample size calculations

The sample size calculation is based on GDM incidence, ${ }^{38}$ which is one of the primary outcomes in our clinical trials. According to previous studies, ${ }^{404}$ the reduction of GDM occurrence is expected to be $50 \%$ that is, an estimated detection rate of $20 \%$ in the control group and $11 \%$ in the experimental group. For $80 \%$ power and a two-sided $\alpha$ level of 0.05, 253 participants are required in each group. With estimated $20 \%$ attrition in the analysis, we will recruit 300 participants in each group, for a total number of 600 .

\section{Data analysis}

Baseline demographic characteristics of both mothers and infants will be presented as mean (SD) or median for continuous variables and count (per cent) for categorical variables. ${ }^{42}$ The results of the logistic regression will be expressed as associated $p$ values for hypothesis testing and ORs. For all tests, alpha $=0.05$ will be regarded as the level of significance. For mothers, comparisons will be performed between groups taking different supplements and subgroups stratified by BMI. For neonates, comparisons with the same grouping criteria will be carried out to determine differences in microbiome profiles as well as growth and development.

For microbiome profile comparisons, the number of OTUs and the Shannon index or Simpson index will be used to estimate the richness and diversity. Beta diversity including weighted and unweighted Unifrac Distance will be used to assess potential clusters of microbiota composition. By principal component analyses, statistically significant clusters will be identified using Adonis. ${ }^{43}$ For normally distributed data with or without $\mathrm{z}$ transformation, a two-way repeated-measure analysis of variance will be used to compare the differences between groups and the changes over time. Otherwise, a mixed linear model analysis of variance, permutational analysis of variance and other non-parametric tests will be used. ${ }^{44}$

To verify the effects of OS on maternal metabolism, immune status and weight gain, a two-way repeated-measure analysis of variance will be performed to test the differences between major groups or subgroups as well as temporal changes within each group. Student's t test or non-parametric test will be used for parameters at each single timepoint.

For bivariate association analysis, the $\chi^{2}$ test will be used to determine the association between OS exposure and adverse pregnancy outcomes including GDM. For multivariate analysis, logistic regression will be built to model 
the participants' sensitivity to OS by combining microbiome characteristics and basic demographic factors.

For the association between OS exposure and fetal developmental status, the differences in infant height, body weight and head/chest/biceps circumferences at birth and 42 days postpartum will be compared by Student's t test for normally distributed data or a non-parametric test otherwise.

Multiple imputation and direct discarding will be used for handing small and large missing data, respectively. The statistical analysis will be performed using the SAS platform (V.9.4, SAS, USA) and bioinformatics analysis for microbiome will use R software (Bell Laboratories, formerly AT\&T, now Lucent Technologies).

\section{Patient and public involvement}

The patients and the public will not be involved in the design, assessment or conduction of this trial. Recruitment information is available on a social networking application (ie, WeChat) and in our hospital. This information will allow pregnant women to contact researchers if they are willing to participant. Women with adverse outcomes will be followed up throughout their pregnancy and will be referred to experts. We will report study results and conclusions to participants in plain language after the entire trial is completed.

\section{DISCUSSION}

Through broad modification of gut microbiota, prebiotics might improve immune functions and correct disturbances in lipid and glucose metabolism. In this prospective, double-blinded, randomised study with a large sample size, we will explore the health-promoting effects of an OS compound. More systematic information can be extracted from multidimensional data with various statistical analyses, thus providing evidence of direct and indirect effects of OS supplementation during pregnancy in a Chinese population.

We will stratify pregnant women into underweight, normal, overweight and obese categories. The rationale is that the association of gut microbiota and GDM incidence varies with different BMIs. Thus, we assume that a specific population may benefit more from current intervention. OS supplementation will cover the whole pregnancy, and the follow-up will extend until 42 days after delivery, so that long-term effects can be further explored on the neonatal gut microbiota, as well as the growth and development of infants.

There are several limitations in this study. First, due to the lack of previous experimental data, an accurate sample size to reflect the effects of OS compound on microbiome is difficult to estimate. Previous RCT research verified that exercise could significantly lower incidence of GDM $(22.0 \%$ vs $40.6 \%) .{ }^{40}$ Another study that examined the effects of probiotics (Lactobacillus rhamnosus GG and Bifidobacterium lactis Bb12) or placebo under intensive diet counselling reported a $67 \%$ risk reduction. ${ }^{41}$ Thus, we set $50 \%$ reduction rate in GDM incidence, which may be lower in a real-world setting. Second, with limited knowledge of pharmacodynamics of prebiotics, further studies on the metabolism of OS should be performed after validation. Third, as prebiotics cannot be digested by the human gut, excessive prebiotics can induce bloating, osmotic diarrhoea and disturbances in electrolytes and acid-base balance, resulting in a reduced follow-up duration. ${ }^{45}$ Last but not least, although questionnaires designed to track dietary habits and physical activity will be administered, multiple lifestyle factors could influence the composition of the microbiota structure and could act as confounders. ${ }^{46} 47$

Based on the validation of beneficial effects of OS preparation on the microbiome and pregnancy outcomes and its exclusive preventive roles in overweight and obese pregnant women, OS could be suggested as a nutrient supplement during pregnancy.

\section{ETHICS AND DISSEMINATION}

This clinical trial was approved by PUFH Clinical Trial Ethics Committee. The study design, ethics and safety information went through thorough examination. The trial was registered on www.chictr.org.cn. The investigators have the right to publish and are responsible for the results. All the collaborators are contributing significantly to the research, and the successful publication is credited to them equally. At the end of this study, we are expected to publish one or more scientific manuscripts in peer-reviewed journals. We also plan to present our results at national and international conferences through oral or poster presentations for further dissemination.

\section{DATA MONITORING}

The data are stored on h6world (a widely used data storage platform ${ }^{48}$ and monitored by the School of Public Health, Peking University and PUFH Clinical Trial Ethics Committee. The data monitoring committee is independent from the sponsor and has no competing interests, and it will ensure that data management will be conducted according to this protocol. The trial procedure will be audited annually by the Beijing Natural Science Foundation and PUFH Clinical Trial Ethics Committee, independent of investigators.

\section{AVAILABILITY OF DATA AND MATERIAL}

All aggregated data, including interim outcomes of the clinical trial, will be accessible to the research team and can be accessed from Chinese clinical trial registry within 6 months after the whole study is finished. The raw data used in this study can be accessed by contacting corresponding authors. The participant-level data will be entered into secure databases protected by passwords, with access restricted to only investigators. The data will be disclosed to participants after the completion of data analysis. No additional data are available. 
Acknowledgements The authors would like to thank all the participants in this study, the Beijing Natural Science Foundation-San Yuan Joint Research Fund for providing technical support and the School of Public Health, Peking University Health Science Centre for providing support in data analysis. We would also like to thank the patient adviser for contributing to the trial by providing the conditions of each patient.

Collaborators Professor Lin An from School of Public Health, Peking University.

Contributors SW, RP, YL, SQ, HY and JM conceived the study design. SW and RP drafted and revised the manuscript. HY and JM helped in developing the protocol and will supervise the overall study conduct. All authors provided critical input to the manuscript.

Funding This work was supported by the Beijing Natural Science Foundation (S170002) and the National Key Technologies R\&D programme of China (2016YFC1000303). This study was designed independently and will be conducted and interpreted by our research team without interference from any other entity.

Competing interests None declared.

Patient consent for publication Not required.

Ethics approval The experiment was approved and supervised by PUFH, National Unit of Clinical Trial Ethics Committee. We will report to ethics committee if any adverse outcomes occur and will submit safety reports twice a year. In addition, protocol amendments will be reported and communicated with journal editors and corresponding revisions will be made in clinical trial registration.

Provenance and peer review Not commissioned; externally peer reviewed. Data availability statement Data are available on reasonable request.

Open access This is an open access article distributed in accordance with the Creative Commons Attribution Non Commercial (CC BY-NC 4.0) license, which permits others to distribute, remix, adapt, build upon this work non-commercially, and license their derivative works on different terms, provided the original work is properly cited, appropriate credit is given, any changes made indicated, and the use is non-commercial. See: http://creativecommons.org/licenses/by-nc/4.0/.

\section{REFERENCES}

1. Caricilli AM, Saad MJA. Gut microbiota composition and its effects on obesity and insulin resistance. Curr Opin Clin Nutr Metab Care 2014;17:312-8.

2. Zhao L, Zhang F, Ding $X$, et al. Gut bacteria selectively promoted by dietary fibers alleviate type 2 diabetes. Science 2018;359:1151-6.

3. Wu H, Esteve E, Tremaroli V, et al. Metformin alters the gut microbiome of individuals with treatment-naive type 2 diabetes, contributing to the therapeutic effects of the drug. Nat Med 2017;23:850-8.

4. Koren O, Goodrich JK, Cullender TC, et al. Host remodeling of the gut microbiome and metabolic changes during pregnancy. Cell 2012;150:470-80.

5. Neuman $\mathrm{H}$, Koren $\mathrm{O}$. The pregnancy microbiome. Nestle Nutr Inst Workshop Ser 2017;88:1-9.

6. Kuang Y-S, Lu J-H, Li S-H, et al. Connections between the human gut microbiome and gestational diabetes mellitus. Gigascience 2017;6:1-12.

7. Gomez-Arango LF, Barrett HL, Mclntyre HD, et al. Increased systolic and diastolic blood pressure is associated with altered gut microbiota composition and butyrate production in early pregnancy. Hypertension 2016;68:974-81.

8. Kindinger LM, Bennett PR, Lee YS, et al. The interaction between vaginal microbiota, cervical length, and vaginal progesterone treatment for preterm birth risk. Microbiome 2017;5

9. Garcia-Mantrana I, Collado MC. Obesity and overweight: impact on maternal and milk microbiome and their role for infant health and nutrition. Mol Nutr Food Res 2016;60:1865-75.

10. Tilg $\mathrm{H}$, Adolph TE. Influence of the human intestinal microbiome on obesity and metabolic dysfunction. Curr Opin Pediatr 2015;27:496-501.

11. Rosenbaum M, Knight $R$, Leibel RL. The gut microbiota in human energy homeostasis and obesity. Trends Endocrinol Metab 2015;26:493-501.

12. Thaiss CA, Zmora N, Levy M, et al. The microbiome and innate immunity. Nature 2016;535:65-74.

13. Gensollen T, lyer SS, Kasper DL, et al. How colonization by microbiota in early life shapes the immune system. Science 2016;352:539-44.
14. Rutayisire E, Huang K, Liu Y, et al. The mode of delivery affects the diversity and colonization pattern of the gut microbiota during the first year of infants' life: a systematic review. BMC Gastroenterol 2016;16:86

15. Barrett HL, Dekker Nitert M, Conwell LS, et al. Probiotics for preventing gestational diabetes. Cochrane Database Syst Rev $2014 ; 152$.

16. Laitinen K, Poussa T, Isolauri E, et al. Probiotics and dietary counselling contribute to glucose regulation during and after pregnancy: a randomised controlled trial. Br J Nutr 2009;101:1679-87.

17. Nitert MD, Barrett HL, Foxcroft K, et al. Spring: an RCT study of probiotics in the prevention of gestational diabetes mellitus in overweight and obese women. BMC Pregnancy Childbirth 2013;13:50.

18. Callaway LK, Mclntyre HD, Barrett HL, et al. Probiotics for the prevention of gestational diabetes mellitus in overweight and obese women: findings from the spring double-blind randomized controlled trial. Diabetes Care 2019;42:dc182248-71.

19. Gibson GR, Hutkins $R$, Sanders ME, et al. Expert consensus document: the International scientific association for probiotics and prebiotics (ISAPP) consensus statement on the definition and scope of prebiotics. Nat Rev Gastroenterol Hepatol 2017;14:491-502.

20. Gibson GR, Probert HM, Loo JV, et al. Dietary modulation of the human colonic microbiota: updating the concept of prebiotics. Nutr Res Rev 2004;17:259-75.

21. Roberfroid MB. Prebiotics and probiotics: are they functional foods? Am J Clin Nutr 2000;71:1682S-7. discussion 88S-90S.

22. Bouhnik Y, Flourié B, D'Agay-Abensour L, et al. Administration of transgalacto-oligosaccharides increases fecal bifidobacteria and modifies colonic fermentation metabolism in healthy humans. J Nutr 1997;127:444-8.

23. Hidalgo-Cantabrana C, Delgado S, Ruiz L, et al. Bifidobacteria and their health-promoting effects. Microbiol Spectr 2017;5.

24. Vulevic J, Drakoularakou A, Yaqoob P, et al. Modulation of the fecal microflora profile and immune function by a novel transgalactooligosaccharide mixture (B-GOS) in healthy elderly volunteers. Am J Clin Nutr 2008;88:1438-46.

25. Vulevic J, Juric A, Walton GE, et al. Influence of galactooligosaccharide mixture (B-GOS) on gut microbiota, immune parameters and metabonomics in elderly persons. Br J Nutr 2015;114:586-95.

26. McLoughlin RF, Berthon BS, Jensen ME, et al. Short-Chain fatty acids, prebiotics, synbiotics, and systemic inflammation: a systematic review and meta-analysis. Am J Clin Nutr 2017;106:930-45.

27. Vinolo MAR, Rodrigues HG, Nachbar RT, et al. Regulation of inflammation by short chain fatty acids. Nutrients 2011;3:858-76.

28. Waldecker M, Kautenburger $\mathrm{T}$, Daumann $\mathrm{H}$, et al. Inhibition of histone-deacetylase activity by short-chain fatty acids and some polyphenol metabolites formed in the colon. J Nutr Biochem 2008;19:587-93.

29. O'Connor S, Chouinard-Castonguay S, Gagnon C, et al. Prebiotics in the management of components of the metabolic syndrome. Maturitas 2017;104:11-18.

30. Sharma S, Prebiotics PS. And lipid metabolism: a review. Altern Ther Health Med 2015;21(Suppl 3):34-42.

31. Delzenne NM, Williams CM. Prebiotics and lipid metabolism. Curr Opin Lipidol 2002;13:61-7.

32. Wang B, Brand-Miller J. The role and potential of sialic acid in human nutrition. Eur J Clin Nutr 2003;57:1351-69.

33. Charbonneau MR, O'Donnell D, Blanton LV, et al. Sialylated milk oligosaccharides promote microbiota-dependent growth in models of infant undernutrition. Cell 2016;164:859-71.

34. Zhou B. Predictive values of body mass index and waist circumference to risk factors of related diseases in Chinese adult population]. Zhonghua Liu Xing Bing Xue Za Zhi 2002;23:5-10.

35. WHO Expert Consultation. Appropriate body-mass index for Asian populations and its implications for policy and intervention strategies. Lancet 2004;363:157-63.

36. Lewis ZT, Davis JCC, Smilowitz JT, et al. The impact of freezedrying infant fecal samples on measures of their bacterial community profiles and milk-derived oligosaccharide content. PeerJ 2016;4:e1612.

37. Gai HH, Sun Y, Fan YL, et al. A survey on prevalence of breastfeeding and relative factors in a community of Beijing. Chin $J$ Soc Med 2018;35:612-4.

38. Wei YM, Yang HX, Zhu WW, et al. Risk of adverse pregnancy outcomes stratified for pre-pregnancy body mass index. J Matern Fetal Neonatal Med 2016;29:2205-9. 
39. Metzger BE, Gabbe SG, Persson B, et al. International association of diabetes and pregnancy study groups recommendations on the diagnosis and classification of hyperglycemia in pregnancy: response to Weinert. Diabetes Care 2010;33:e98-82.

40. Wang $\mathrm{C}$, Wei $\mathrm{Y}$, Zhang $\mathrm{X}$, et al. A randomized clinical trial of exercise during pregnancy to prevent gestational diabetes mellitus and improve pregnancy outcome in overweight and obese pregnant women. Am J Obstet Gynecol 2017;216:340-51.

41. Luoto R, Laitinen K, Nermes M, et al. Impact of maternal probioticsupplemented dietary counselling on pregnancy outcome and prenatal and postnatal growth: a double-blind, placebo-controlled study. Br J Nutr 2010;103:1792-9.

42. Simioni J, Hutton EK, Gunn E, et al. A comparison of intestinal microbiota in a population of low-risk infants exposed and not exposed to intrapartum antibiotics: The Baby \& Microbiota of the Intestine cohort study protocol. BMC Pediatr 2016;16:183.

43. Sindhu KNC, Cunliffe N, Peak M, et al. Impact of maternal antibodies and infant gut microbiota on the immunogenicity of rotavirus vaccines in African, Indian and European infants: protocol for a prospective cohort study. BMJ Open 2017;7:e016577.

44. Crusell MKW, Hansen TH, Nielsen T, et al. Gestational diabetes is associated with change in the gut microbiota composition in third trimester of pregnancy and postpartum. Microbiome 2018;6.

45. Marteau P, Seksik P. Tolerance of probiotics and prebiotics. J Clin Gastroenterol 2004;38(6 Suppl):S67-9.

46. Ussar S, Griffin NW, Bezy O, et al. Interactions between gut microbiota, host genetics and diet modulate the predisposition to obesity and metabolic syndrome. Cell Metab 2015;22:516-30.

47. Kang SS, Jeraldo PR, Kurti A, et al. Diet and exercise orthogonally alter the gut microbiome and reveal independent associations with anxiety and cognition. Mol Neurodegener 2014;9:36.

48. Lin J-Y, Huang Y, Su Y-A, et al. Association between perceived Stressfulness of stressful life events and the suicidal risk in Chinese patients with major depressive disorder. Chin Med J 2018;131:912-9. 